TI 2011-015/1

Tinbergen Institute Discussion Paper

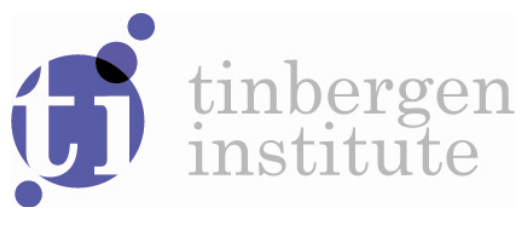

\title{
Capacity Constraints and Beliefs about Demand
}

Nick Vikander

Tinbergen Institute, Erasmus University Rotterdam, and University of Edinburgh. 
Tinbergen Institute is the graduate school and research institute in economics of Erasmus University Rotterdam, the University of Amsterdam and VU University Amsterdam.

More TI discussion papers can be downloaded at http://www.tinbergen.nl

Tinbergen Institute has two locations:

Tinbergen Institute Amsterdam

Gustav Mahlerplein 117

1082 MS Amsterdam

The Netherlands

Tel.: +31(0)205251600

Tinbergen Institute Rotterdam

Burg. Oudlaan 50

3062 PA Rotterdam

The Netherlands

Tel.: +31(0)10 4088900

Fax: +31(0)104089031

Duisenberg school of finance is a collaboration of the Dutch financial sector and universities, with the ambition to support innovative research and offer top quality academic education in core areas of finance.

DSF research papers can be downloaded at: http://www.dsf.nl/

Duisenberg school of finance

Gustav Mahlerplein 117

1082 MS Amsterdam

The Netherlands

Tel.: +31(0)20 5258579 


\title{
Capacity Constraints and Beliefs About Demand
}

\author{
Nick Vikander*
}

November 2010

\begin{abstract}
This paper examines how a firm can strategically choose its capacity to manipulate consumer beliefs about aggregate demand. It looks at a market with social effects where consumers want to do what is popular, to buy what they believe others want to buy. By imposing a capacity constraint and setting a price just low enough for it to bind, the firm can fool certain naive consumers into believing that demand is greater than it actually is. This will in turn increase the willingness to pay of all consumers through social effects. In equilibrium, the firm will impose a capacity constraint whenever demand is lower than expected, even when the number of naive consumers is arbitrarily small. (JEL D80, L00)
\end{abstract}

\section{Introduction}

Firms looking to build a favorable buzz around their products can often benefit by selling out. Dining at a particular restaurant or attending a Broadway show may be more attractive if the restaurant is usually full, or the show difficult to get into. Concert promoters putting new tickets on sale will often draw attention to a string of past sold-out performances. ${ }^{1}$ In professional

*Tinbergen Institute and Erasmus University Rotterdam, University of Edinburgh, nick.vikander@ed.ac.uk, www.tinbergen.nl/ vikander

${ }^{1}$ For an example with Bruce Springsteen and the E Street Band, see www.shorefire.com/index.php? $\mathrm{a}=$ pressreleaseo $=83$ 
sports, fans actively discuss and compare the consecutive sell-outs streaks of different teams. ${ }^{2}$ The Boston Red Sox marked the occasion of 600 straight sell-outs at Fenway Park with a widely publicized ceremony, where principal owner John W. Henry threw 600 commemorative baseballs into the crowd. ${ }^{3}$

The desire to generate a positive buzz through sell-outs can cause firms to act strategically. Palm is reported to have stocked low numbers of its Pre phone to enhance the allure of the product, with analysts commenting that selling out would be seen as an important success. ${ }^{4}$ Some firms have been accused of deliberately trying to mislead consumers, using perceived shortages as a shrewd marketing tactic. There were widespread claims that Nintendo deliberately produced too few of its new, widely popular Wii console in 2006 and 2007, in order to artificially increase demand. ${ }^{5}$ The Red Sox have also been accused of trying to artificially sustain their sell-out streak, by selling tickets to secondary sellers who are willing to bear more risk. Games are then officially sold out even if some tickets never find their way into the hands of fans. This keeps the sell-out streak alive, which is viewed as a great selling point for the team. ${ }^{6}$

These examples all suggest that firms may strategically restrict capacity to manipulate consumer beliefs about aggregate demand. However, this issue does not seem to have received any attention up until now in the economics literature. One potential reason could be the difficulty of reconciling this idea with the common view that, in equilibrium, consumer beliefs should be correct. By taking a somewhat different approach involving bounded rationality, this paper aims to fill this gap.

I look at a market where consumers care about the popularity of what they buy. Consumers are willing to pay more for a good if they believe it is a hit, something many others want to buy as well. Whether it is wearing clothes of a certain brand name, going to a particular performance at the theater, or attending a concert or sporting event, these consumers care about whether something is the "in" thing to do. I refer to this consumption externality as a social effect.

I assume consumers can observe quantity sold but not quantity demanded,

\footnotetext{
${ }^{2}$ For an example in professional hockey, see http://hfboards.com/showthread.php?t=477736\&page=2

${ }^{3}$ See "The Red Sox nurture a 'Sellout' Streak", BusinessWeek, July 29, 2010

${ }^{4}$ www.bloomberg.com/apps/news?pid=newsarchive\&sid=az5nzqH0Mt4M\&refer $=$ us

${ }^{5}$ www.cio.com/article/445316/Nintendo_Wii_Shortage_Shrewd_Marketing_or_Flawed_Supply_Chain_

${ }^{6}$ www.bloomberg.com/news/2010-07-30/red-sox-s-ticket-policy-keeps-sellout-streakalive-with-help-of-resellers.html
} 
which means that a binding capacity constraint can influence their beliefs about a good's popularity. In the setting I examine, a binding capacity constraint will cause some consumers to infer that demand is higher than it actually is. These consumers do not take into account that the firm is informed about demand, and has an incentive to influence their beliefs. I refer to these consumers as naive, and the mechanism I explore depends only on their numbers being strictly positive.

Specifically, I consider a two period game where the firm is fully informed about the total number of consumers in the market, but some consumers are not. Each consumer's willingness to pay is increasing in his expectation of quantity demanded, and a strictly positive fraction of consumers are naive. Naive consumers are rational and update their beliefs about demand using Bayes' rule, except they do not directly condition these beliefs on the firm's equilibrium strategy.

The firm sets prices and also decides whether to impose a capacity constraint, which if imposed must remain in place for both periods. I show that the firm can use a capacity constraint in period 1 in order to increase demand in period 2. If the firm imposes a capacity constraint, it will set a period 1 price such that the constraint exactly binds. Naive consumers see that the firm has sold out, but do not realize that excess demand is actually zero. Instead, they form an expectation about the amount of excess demand, reasoning that quantity demanded must be greater than or equal to quantity sold. The mistaken beliefs of naive consumers will then increase the willingness to pay of all consumers in period 2 through social effects.

In particular, the firm will impose a capacity constraint whenever demand is lower than expected, but it will not impose a capacity constraint when demand is sufficiently high. ${ }^{7}$ The firm faces a trade-off, since a binding capacity constraint will increase period 2 demand beyond what it otherwise would have been, but will also leave the firm unable to expand output above its period 1 level. When demand is lower than expected, the firm will impose a capacity constraint because, in its absence, expanding output in period 2 would not have been an issue. When demand is high, the firm will not impose a constraint because there is little benefit to fooling naive consumers.

I also show that if the firm does impose a capacity constraint, then naive consumers never discover that they have been fooled. Moreover, the results

\footnotetext{
${ }^{7}$ Throughout the paper, I will write "lower than expected" to mean lower than the ex-ante expectation of both the firm and of consumers.
} 
continue to hold when social effects are weak, or when the number of naive consumers is arbitrarily small.

The view that social effects can generate consumption externalities goes back many years, and includes Leibenstein (1950) and Becker (1974). Leibenstein examined how individual demand may be increasing in aggregate demand, and termed this the bandwagon effect. The bandwagon effect is related to work on network goods, such as Katz and Shapiro (1985), where the eventual surplus from buying depends on the total number of consumers who also buy. With social effects, however, the externality should be directly related to a good's popularity, not its sales. What matters is not how many people actually buy, which may be limited by rationing, but how many people would like to buy. Becker (1991) and Karni and Levin (1994) follow this route, and assume willingness to pay is increasing in quantity demanded, not quantity sold. Basu (1987) takes a related approach, where willingness to pay depends directly on excess demand.

If the bandwagon effect were modeled in terms of informational cascades, as in Bikhchandani, Hirshleifer, and Welch (1992) and Banerjee (1992), then the relevant issue would again be consumers' expectation of demand, not necessarily of sales. A consumer who knows that others have private information about quality is interested in discovering how many people would like to buy the good, regardless of whether rationing prevents them from actually doing so. ${ }^{8}$

A key assumption of the paper is that consumers can directly observe quantity sold, but not quantity demanded; when quantity sold equals capacity, they cannot observe the extent of excess demand. This assumption is more reasonable in some settings than in others. It may be easier to see the number of people standing in the cue outside a nightclub than to see the number of people inside. In contrast, a person glancing into a restaurant may observe that the tables are full, without getting a good sense of how many people have been turned away for lack of space. Similarly, people can easily check the number of tickets sold for major sporting events, as these figures are consistently reported in the press. It is more difficult to find precise information on the number of people who tried to buy tickets, but were unable to do so.

Various papers have explored how a firm can benefit from rationing, ei-

\footnotetext{
${ }^{8}$ This distinction would only be relevant in a model combining informational cascades and rationing, something which has yet to be examined.
} 
ther by imposing a capacity constraint or setting a price that creates excess demand. One important difference is that none of these papers look at how restricting capacity can influence consumer beliefs. Another is that they identify benefits to actually having excess demand, whereas I show the benefit of having naive consumers mistakenly believe there is excess demand.

DeSerpa and Faith (1996) argue rationing can be useful if serving consumers with a low intrinsic valuation generates positive externalities. For example, many people appreciate loud cheering at a sporting event, but those who tend to cheer the most may be unable to pay a high ticket price. A random rationing rule then allows some of these low valuation consumers to be served, which increases the willingness to pay of others. DeGraba (1995) shows that the threat of future rationing can convince consumers to buy early, which can increase profits if valuations become more heterogeneous over time. Nocke and Peitz (2007) also look at the threat of future rationing, and show how it can induce high valuation consumers to separate and purchase before low valuation consumers. Denicolo and Garella (1999) consider a durable goods monopoly, and show excess demand can be optimal if the rationing rule is not efficient. Rationing then effectively shifts some high valuation consumers from earlier to later periods, smoothing demand over time and helping the firm commit to a higher price.

The paper in this literature that is closest to the current work is Becker (1991). Becker also assumes that there are social effects to consumption, and that willingness to pay is increasing in aggregate quantity demanded. He shows that if social effects are strong enough to make aggregate demand upwards sloping, then a capacity constrained monopolist may choose to have excess demand. Marginally increasing the price could then cause demand to collapse to zero.

Although Becker considers the same social effects, many other important features of the model are different. He looks at a one shot game, where the results rely both on demand being upwards sloping and on the capacity constraint being exogenous. There is no uncertainty, so influencing beliefs plays no role. In contrast, I assume that the capacity constraint is endogenous, and it is the presence of naive consumers, not any upwards sloping demand, which drives the results. As noted above, there are also important differences in the results themselves.

The idea that some consumers do not fully understand how other people's equilibrium actions depend on their private information is also quite reasonable. The approach I take is related to that of cursed equilibrium, as 
in Eyster and Rabin (2005). It also follows in a recent strand of literature in industrial organization looking at how firms can hide information from boundedly rational consumers. I comment more on how this approach to naive consumers relates to the literature in Section 4.

The rest of the paper is organized as follows. Section 2 describes the model. Section 3 contains the analysis, and discusses the intuition for the results. Section 4 presents some of the related literature dealing with naive consumers, and Section 5 concludes.

\section{The Model}

A monopolist produces a homogeneous good at constant marginal cost, normalized to zero. It faces a market of consumers who have unit demand in each of two periods, where the discount factor is $\delta \in(0,1]$.

The total number of consumers in the market is known to the firm, but not to all consumers. There is a mass $M$ of potential consumers, but the actual mass of consumers in the market is $x M$, where $x$ is a draw of a random variable $X$ distributed according to $F$ with full support on $(0,1] .^{9}$

Each consumer's willingness to pay for the good consists of two parts, intrinsic utility and social utility. A consumer's intrinsic utility from buying is an independent draw from a uniform distribution on $\left[-A_{0}, A\right]$, where $A_{0}>$ $0, A>0$ and $A_{0}+A=M .^{10}$

A consumer's social utility from buying is $\mathrm{Cq}(\mathrm{p}, \mathrm{x})$, where $C<1$ is a strictly positive constant and $q(p, x)$ is aggregate quantity demanded at price $\mathrm{p}$ when $X=x$. If a consumer does not buy the good, then his utility in that period is zero. A consumer's willingness to pay is equal to his expected utility from buying, which is his intrinsic utility plus $C E[q(p, X)]$. Here, $E[q(p, X)]$ is the consumer's expectation of quantity demanded taken over $X$.

There are two types of consumers: a fraction $1-\alpha$ are informed and sophisticated, while a fraction $\alpha$ are uninformed and naive, with $\alpha \in(0,1]$.

\footnotetext{
${ }^{9}$ The mechanism should apply equally well if there was uncertainty about other consumers' preferences. However, modeling uncertainty in terms of the number of consumers in the market has two practical advantages. It means a consumer cannot infer anything about aggregate demand from his own willingness to pay. It also means that social effects will just change the slope of the inverse demand function and not its intercept, which simplifies the analysis.

${ }^{10}$ The assumption that intrinsic utility can be negative is simply to avoid corner solutions after taking into account social effects, as explained below.
} 
Informed and sophisticated consumers observe the value of $x$ and are fully rational, in the standard sense. Uninformed and naive consumers just know how $X$ is distributed. They are rational, except they do not take into account how the firm's equilibrium strategy may be related to its private information about $x$. Naive consumers update their beliefs using Bayes' rule, by reasoning what values of $x$ are consistent with the quantity sold they observe. However, they do not condition these beliefs directly on the firm's strategy. In short, the firm cannot signal to naive consumers. Intrinsic utility is unrelated to consumers' information or degree of sophistication, so that the intrinsic utility of both types of consumers is uniformly distributed on $\left[-A_{0}, A\right]{ }^{11}$

The game proceeds as follows. In period 0 , nature draws a single value of $x$ which is observed by the firm and by informed consumers. This draw determines the number of consumers in the market, which is constant across periods. In period 1, the firm decides whether to impose a capacity constraint and, if so, chooses a value of $K>0$. If the firm imposes a capacity constraint with a given $K$, then quantity sold cannot exceed $K$ in either period: $Q_{t} \leq K$, for $t \in\{1,2\}$. The firm also sets a period 1 price, $p_{1}$.

Each consumer observes $p_{1}$ and $K$ if there is a capacity constraint, and decides whether to buy a unit of the good. Aggregate quantity demanded is then $q_{1}$. If $q_{1}>K$, then a mass $K$ of consumers are allocated the good according to some rationing rule, the details of which are unimportant for the results. After consumers buy the good, they observe $Q_{1}$ but not $q_{1}$, and naive consumers update their beliefs about $X$. The firm earns period 1 profits $\pi_{1}$.

In period 2 , the firm sets price $p_{2}$, and each consumer again decides whether to buy a unit of the good. This gives aggregate quantity demanded $q_{2}$. As before, if $q_{2}>K$, then a mass $K$ of consumers are allocated the good according to the rationing rule. The firm earns period 2 profits $\pi_{2}$, and the game ends.

The firm chooses the strategy which maximizes profits, where I assume it will only impose a capacity constraint if doing so yields strictly higher profits. This will mean I need not consider situations where the firm is indifferent between not setting a capacity constraint, and setting a constraint with such a high value of $K$ that it will never bind.

The assumption $C<1$ will imply that the demand curve is downward

\footnotetext{
${ }^{11} \mathrm{~A}$ more general treatment would also include consumers who are uninformed but sophisticated. This would introduce the possibility of signaling, which would complicate the analysis. Assuming there are no such consumers allows me to concentrate on the main issue of the paper, which is the firm's incentive to manipulate naive consumers.
} 
sloping. While this is not essential for the analysis, it is necessary to have interior solutions. With a uniform distribution of intrinsic utility, $C \geq 1$ would imply that the firm always chooses to sell to all consumers in the market. Downward sloping demand also means that there is no issue of multiple equilibria. The assumption $C<1$ sets the analysis firmly apart from Becker (1991), whose results depend on demand being upwards sloping on at least some interval.

To guarantee interior solutions, the intrinsic utility of the consumer who is least willing to buy must be sufficiently negative. If all $M$ consumers are expected to buy, then the willingness to pay of the consumer with the lowest intrinsic utility is $-A_{0}+C M$. I assume that

$$
\left(\frac{C}{1-C}\right) A \leq A_{0}
$$

which ensures that this consumer's willingness to pay is never greater than zero. The assumption $M=A_{0}+A$ ensures that $A_{0}$ and $M$ play no further role in the analysis.

In terms of notation, I use a number of different subscripts. The first subscript refers to period $t \in\{1,2\}$. The second subscript refers to whether the firm has imposed a capacity constraint $c$, or not, $u$, which stand for "constrained" and "unconstrained". For period $t$, I use $q_{t c}$ for quantity demanded, $Q_{t c}$ for quantity sold, and $\pi_{t c}$ for profits if the firm has imposed a capacity constraint, and I use $q_{t u}, Q_{t u}$, and $\pi_{t u}$ if the firm has not. I abuse notation and also use $q_{t c}$ and $q_{t u}$ to refer to the demand function in period $t$. Finally, I sometimes use the subscripts $N$ and $S$ to refer to demand from naive and sophisticated consumers.

\section{Analysis}

I first show how aggregate demand in a given period will depend on the expectations of naive consumers. Because of social effects, the willingness to pay of naive consumers is increasing in their expectation of quantity demanded. The only thing uncertain is the number of consumers in the market, so willingness to pay can be expressed as a function of naive consumers' expectation about $X$. I denote this expectation by $E_{X}$, and show how it affects aggregate demand. 
The notation $E_{X}$ distinguishes this from naive consumers' ex-ante expectation about $X$, which I denote by $E[X]$. These expectations will coincide in period 1 , but not in period 2 after naive consumers update their beliefs.

I let $q_{N}$ denote the demand of naive consumers, and $q_{S}$ the demand of sophisticated consumers. Aggregate demand is then $q_{N}+q_{S}$.

The quantity demanded by naive consumers is equal to their number in the market, $\alpha x M$, multiplied by the fraction of these consumers for whom the utility of buying exceeds the price. Naive consumers do not know the value of $x$, so that buying gives expected social utility $C E\left[q_{N}+q_{S}\right]$. Intrinsic utility is uniformly distributed on $\left[-A_{0}, A\right]$, which means that the fraction of naive consumers who will buy is $\left(A+C E\left[q_{N}+q_{S}\right]-p\right) /\left(A+A_{0}\right)$. The denominator equals $M$ by assumption, so that demand from naive consumers is

$$
q_{N}=\alpha\left(A+C E\left[q_{N}+q_{S}\right]-p\right) x .
$$

The demand from sophisticated consumers is given by a similar expression, where $\alpha$ is replaced by $(1-\alpha)$. An important difference is that naive consumers know the value of $x$, so their social utility is $C\left(q_{N}+q_{S}\right)$, rather than its expectation.

$$
q_{S}=(1-\alpha)\left(A+C\left(q_{N}+q_{S}\right)-p\right) x .
$$

To see how aggregate demand depends on $E_{X}$, I need to express $E\left[q_{N}\right]$ and $E\left[q_{S}\right]$ in terms of $E_{X}$. That is, I need to show how the expectation of naive consumers about their own quantity demanded, and that of sophisticated consumers, depends on their expectation about the number of consumers in the market. To do so, I take the expectation of both sides of (1) and (2). This gives

$$
\begin{gathered}
E\left[q_{N}\right]=\alpha\left(A+C E\left[q_{N}\right]+C E\left[q_{S}\right]-p\right) E_{X}, \\
E\left[q_{S}\right]=(1-\alpha)\left(A+C E\left[q_{N}\right]+C E\left[q_{S}\right]-p\right) E_{X} .
\end{gathered}
$$

Solving this system of two linear equations in two unknowns yields

$$
E\left[q_{N}\right]=\frac{\alpha E_{X}}{1-C E_{X}}(A-p),
$$




$$
E\left[q_{S}\right]=\frac{(1-\alpha) E_{X}}{1-C E_{X}}(A-p)
$$

The expectation of naive consumers about quantity demanded is increasing in their expectation of $X$. The two expressions only differ in terms of the coefficient $\alpha$ or $1-\alpha$, the proportion of each type of consumer in the market.

I can now substitute these expressions back into (1), to express the demand of naive consumers in terms of $E_{X}$

$$
q_{N}=\alpha x\left(\frac{1}{1-C E_{X}}\right)(A-P) .
$$

Substituting (3) into (2) and solving for $q_{S}$ gives the demand of sophisticated consumers in terms of $E_{X}$

$$
q_{S}=(1-\alpha) x\left[\frac{1}{1-(1-\alpha) C x}\right]\left[1+\left(\frac{\alpha C x}{1-C E_{X}}\right)\right](A-p) .
$$

Aggregate demand is then given by $q_{N}+q_{S}$. In the absence of social effects, $C=0$, aggregate demand simplifies to $x(A-p)$. As expected, this is just the number of consumers in the market whose intrinsic utility from buying exceeds the price.

Looking at (3) and (4) shows that social effects do increase demand, but not by shifting up the demand curve. Regardless of the value of $C$ or $E_{X}$, the firm will not make any sales if it charges a price higher than the intrinsic utility of the top consumer, $p>A$. If the firm did charge such a price, and consumers expected no one to buy, then expected social utility from buying would be zero. It would then indeed be optimal for no consumer to buy. Instead, social effects increase demand by flattening out the demand curve, reducing the magnitude of its slope.

Setting $E_{X}=x$ or $\alpha=0$ gives the full information case, where all consumers observe the value of $x$. There is then no distinction between the two groups of consumers, and aggregate demand simplifies to $x(A-p) /(1-$ $C x)$. The denominator reflects the increased willingness to pay generated through social effects.

The above expressions for $q_{S}$ and $q_{N},(3)$ and (4), reflect the fact that an increase in naive consumers' expectation about $X$ has both a direct and an indirect effect on aggregate demand. An increase in $E_{X}$ directly increases the expected social utility of naive consumers who buy. This will increase their quantity demanded at any given price. 
The increased demand of naive consumers, combined with social effects, are what create an indirect effect of $E_{X}$ on aggregate demand. Higher demand from naive consumers increases the social utility of all consumers who buy. In particular, this increases demand from sophisticated consumers, which in turn will increase the willingness to pay of naive consumers through social effects.

In this way, social effects create a feedback mechanism through which a small change in $E_{X}$ can trigger a relatively large change in aggregate demand. In particular, this will be the case when $C E_{X}$ is close to 1 .

Now that I have established how aggregate demand depends on consumers' expectations, I turn to the problem faced by the firm. The first proposition considers a situation where the firm does not impose a capacity constraint. It describes the demand the firm will then face in each period, and its optimal price.

Proposition 1. Suppose the firm does not impose a capacity constraint. Then period 1 demand is

$q_{1 u}=\left\{(1-\alpha)\left[\frac{1}{1-(1-\alpha) C x}\right]\left[1+\left(\frac{\alpha C x}{1-C E[X]}\right)\right]+\alpha\left(\frac{1}{1-C E[X]}\right)\right\} x(A-p)$,

and period 2 demand is

$$
q_{2 u}=\frac{x}{1-C x}(A-p) .
$$

The firm will set prices $p_{1}=p_{2}=A / 2$ and earn

$$
\begin{gathered}
\pi_{1 u}=\left\{(1-\alpha)\left[\frac{1}{1-(1-\alpha) C x}\right]\left[1+\left(\frac{\alpha C x}{1-C E[X]}\right)\right]+\alpha\left(\frac{1}{1-C E[X]}\right)\right\} x\left(\frac{A^{2}}{4}\right), \\
\pi_{2 u}=\frac{x}{1-C x}\left(\frac{A^{4}}{4}\right)
\end{gathered}
$$

giving total profits

$$
\pi_{u}=x\left(\frac{A^{2}}{4}\right)\left\{(1-\alpha)\left[\frac{1}{1-(1-\alpha) C x}\right]\left[1+\left(\frac{\alpha C x}{1-C E[X]}\right)\right]+\alpha\left(\frac{1}{1-C E[X]}\right)+\left(\frac{\delta}{1-C x}\right)\right\} .
$$


Proof. In period 1, the expectation of naive consumers about $X$ is just the ex-ante expectation: $E_{X}=E(X)$. From (3) and (4), period 1 demand from the two groups of consumers is then

$$
\begin{gathered}
q_{S}=(1-\alpha) x\left[\frac{1}{1-(1-\alpha) C x}\right]\left[1+\left(\frac{\alpha C x}{1-C E[X]}\right)\right](A-p), \\
q_{N}=\alpha x\left(\frac{1}{1-C E[X]}\right)(A-p) .
\end{gathered}
$$

Total period 1 demand is $q_{1 u}=q_{S}+q_{N}$, given by (5).

Consumers observe period 1 quantity sold, $Q_{1 u}$, and since there is no capacity constraint this must equal $q_{1 u}$. Consumers are then able to infer the exact value of $x$, because (5) is strictly increasing in $x$.

Period 2 demand is then also given by (3) and (4), but with $E_{X}=x$. That is

$$
\begin{gathered}
q_{S}=(1-\alpha) x\left[\frac{1}{1-(1-\alpha) C x}\right]\left[1+\left(\frac{\alpha C x}{1-C x}\right)\right](A-p), \\
q_{N}=\alpha \frac{x}{1-C x}(A-p) .
\end{gathered}
$$

Demand from sophisticated consumers simplifies to

$$
q_{S}=(1-\alpha) \frac{x}{1-C x}(A-p),
$$

so that period 2 demand, $q_{2 u}=q_{S}+q_{N}$, is given by (6).

Both $q_{1 u}$ and $q_{2 u}$ are proportional to $(A-p)$, and marginal costs are zero, so the optimal price is $p_{1}=p_{2}=A / 2$. This implies

$$
\begin{gathered}
Q_{1 u}=\left\{(1-\alpha)\left[\frac{1}{1-(1-\alpha) C x}\right]\left[1+\left(\frac{\alpha C x}{1-C E[X]}\right)\right]+\alpha\left(\frac{1}{1-C E[X]}\right)\right\} x\left(\frac{A}{2}\right) \\
Q_{2 u}=\frac{x}{1-C x}\left(\frac{A}{2}\right)
\end{gathered}
$$

Period 1 profits $\pi_{1 u}$ are therefore given by (7), and period 2 profits $\pi_{2 u}$ by (8). Total profits are then $\pi_{1 u}+\delta \pi_{2 u}$, which is equal to (9). 
In both period 1 and period 2, demand will be the sum of $q_{N}$ and $q_{S}$, given by (3) and (4). The difference in demand between the two periods will depend only on $E_{X}$, because naive consumers will update their beliefs about $X$ based on what they observe in period 1 .

In period 1, naive consumers just have their ex-ante expectation $E[X]$. Consumers know that period 1 quantity demanded depends on $x$ according to (5), and they are able to observe quantity sold, $Q_{1 u}$. The key point is that when the firm does not impose a capacity constraint, then quantity demanded and quantity sold must coincide. Naive consumers can then infer the true value of $x$, and period 2 demand equals the full information case of (3) and (4) with $E[X]=x$.

If quantity demanded is lower than expected, $x<E[x]$, then naive consumers adjust their beliefs downwards after period 1, and demand will decrease in period 2. In contrast, if quantity demanded is higher than expected, $x>E[x]$, then demand will increase from period 1 to period 2 . The fact that demand is linear with maximum willingness to pay $A$, and that marginal cost is zero, explains why the optimal price is constant at $p_{1}=p_{2}=A / 2$.

I now consider the strategy of a firm which decides to impose a capacity constraint. Period 1 demand will be the same as it was without a constraint, $q_{1 c}=q_{1 u}$, given by (5), because naive consumers will still have expectation $E_{X}=E(X)$. However, a capacity constraint will cause period 2 demand to be different. It will do so by preventing naive consumers from inferring the true value of $x$.

A firm which chooses a capacity constraint must decide on its level, $K$, and also on a price in each period. I first show that if the firm imposes a capacity constraint, then it will exactly bind in both periods, and prices will increase over time.

Proposition 2. Let $\pi_{c}$ denote the maximum profits the firm can earn by setting a capacity constraint and optimally choosing $K, p_{1}$ and $p_{2}$. Suppose $x$ takes on a value such that it is profitable to set a capacity constraint: $\pi_{c}>\pi_{u}$, with $\pi_{u}$ given by (9). Then the firm will set $p_{1}$ and $p_{2}$ such that quantity demanded equals capacity: $q_{1 c}=Q_{1 c}=q_{2 c}=Q_{2 c}=K$. Moreover, we will have $p_{1}<A / 2<p_{2}$.

Proof. As in Proposition 1, period 1 demand $q_{1 c}$ is given by (5). Demand is the same as without a capacity constraint, but now quantity sold cannot exceed capacity, $Q_{1 c} \leq K$. This means period 1 profits can be no greater 
than they would be without a capacity constraint: $\pi_{1 c} \leq \pi_{1 u}$, where $\pi_{1 u}$ is given by (7).

Suppose the firm sets $p_{1}$ so that period 1 quantity demanded is strictly less than capacity, $q_{1 c}<K$. Then $q_{1 c}=Q_{1 c}<K$, and by the same argument as in the proof of Proposition 1, naive consumers can infer the exact value of $x$. This means $q_{2 c}$ is given by (6), as it was without a capacity constraint. The only effect of the capacity constraint in period 2 is to restrict quantity sold, $Q_{2 c} \leq K$. Period 2 profits are therefore no greater than they would be without a capacity constraint: $\pi_{2 c} \leq \pi_{2 u}$, with $\pi_{2 u}$ given by (8). But this contradicts $\pi_{c}>\pi_{u}$, so it must be that $q_{1 c} \geq K$, and $Q_{1 c}=K$. That is, the capacity constraint must bind in period 1 .

Consumers then observe that $Q_{1 c}=K$ at the end of period 1, and naive consumers update their beliefs about $x$. From (5), $q_{1 c}$ is strictly increasing in $x$. Naive consumers therefore infer that $x \geq x^{\prime}$, where $x^{\prime}$ is the lowest value of $x$ such that $q_{1 c} \geq K$. That is, $x^{\prime}=x$ would imply $q_{1 c}=K$. Period 2 demand, $q_{2 c}$, is then given by (5), but with $E[X]$ replaced by $E\left[X \mid X \geq x^{\prime}\right]$.

I argue that the firm must set $p_{1}$ so that $q_{1 c}=K$, and hence $x^{\prime}=x$. If instead $q_{1 c}>K$, then there would be strictly positive excess demand. The firm could marginally increase $p_{1}$ and still sell $Q_{1 c}=K$, which would increase $\pi_{1 c}$. This marginal change in price would also change the way that naive consumers update their beliefs. From (5), $q_{1 c}$ is strictly decreasing in $p$ but strictly increasing in $x$, which implies $x^{\prime}$ is increasing in $p$. A marginal increase of $p_{1}$ therefore increases $x^{\prime}$, as long as quantity demanded is still greater than or equal to capacity. This will in turn increase $E\left[X \mid X \geq x^{\prime}\right]$.

Period 2 demand is given by (5) but with $E[X]$ replaced by $E\left[X \mid X \geq x^{\prime}\right]$, so we know that $q_{2 c}$ is increasing in $E\left[X \mid X \geq x^{\prime}\right]$. A marginal increase in $p_{1}$ therefore increases $q_{2 c}$, and the firm can charge a higher price for any given quantity sold. Period 2 profits are now higher as well, which is a contradiction. It therefore follows that the optimal $p_{1}$ must give $q_{1 c}=K$ and $x^{\prime}=x$.

Period 2 demand is then given by (5), but with $E[X]$ replaced by $E[X \mid X \geq$ $x]$.

$$
\begin{gathered}
q_{S}=(1-\alpha) x\left[\frac{1}{1-(1-\alpha) C x}\right]\left[1+\left(\frac{\alpha C x}{1-C E[X \mid X \geq x]}\right)\right](A-p) \\
q_{N}=\alpha x\left(\frac{1}{1-C E[X \mid X \geq x]}\right) x(A-p)
\end{gathered}
$$


$q_{2 c}=\left\{(1-\alpha)\left[\frac{1}{1-(1-\alpha) C x}\right]\left[1+\left(\frac{\alpha C x}{1-C E\left[X \mid X \geq x^{\prime}\right]}\right)\right]+\alpha\left(\frac{1}{1-C E\left[X \mid X \geq x^{\prime}\right]}\right)\right\} x(A-p)$

Period 2 demand, $q_{2 c}$, is proportional to $A-p$. If the firm did not have a capacity constraint in period 2 , then period 2 profits would be given by $\pi_{2}=$ $p_{2} q_{2 c}$. These profits would be quadratic in $p_{2}$, or equivalently quadratic in $q_{2 c}$. The firm would then choose $p_{2}=A / 2$. Denote the resulting unconstrained, optimal quantity sold by $Q_{2}^{\prime}$, which is (12) evaluated at $p_{2}=A / 2$. Of course, the firm may not be able to actually choose $Q_{2 c}=Q_{2}^{\prime}$, since there is the constraint $Q_{2 c} \leq K$.

From Proposition 1, the optimal period 1 price without a capacity constraint is $p_{1}=A / 2$, with quantity sold $q_{1 u}=Q_{1 u}$, given by (10). The unconstrained optimal price is the same in each period, $p=A / 2$. We therefore have $Q_{1 u}<Q_{2}^{\prime}$, since $E[X \mid X \geq x]>E[X]$ implies $q_{1 u}<q_{2 c}$. This means that, fixing naive consumer beliefs at $E[X]$ in period 1 and at $E[X \mid X \geq x]$ in period 2, the firm would like to increases quantity sold over time.

By (5) and $q_{1 c}=K$, the firm with capacity constraint $K$ will set period 1 price

$p_{1}=A-\frac{K}{x}\left\{(1-\alpha)\left[\frac{1}{1-(1-\alpha) C x}\right]\left[1+\left(\frac{\alpha C x}{1-C E[X]}\right)\right]+\alpha\left(\frac{1}{1-C E[X]}\right)\right\}^{-1}$

Period 1 profits are then $p_{1} K$, so

$\pi_{1 c}=A K-\frac{K^{2}}{x}\left\{(1-\alpha)\left[\frac{1}{1-(1-\alpha) C x}\right]\left[1+\left(\frac{\alpha C x}{1-C E[X]}\right)\right]+\alpha\left(\frac{1}{1-C E[X]}\right)\right\}^{-1}$

Define the loss function $\lambda_{1}(K)$ as $\pi_{1 u}-\pi_{1 c}$, given by (7) and (13). It is the loss in period 1 profits from selling quantity $K$ rather than the unconstrained optimum $Q_{1 u}$. The loss function is positive and increasing quadratically in $\left|K-Q_{1 u}\right|$. It takes on a value of zero at $K=Q_{1 u}$, as does its derivative with respect to $K$.

Define the loss function $\lambda_{2}(K)$ in a similar way. It is $\pi_{2 c}-(A / 2) Q_{2}^{\prime}$, so the loss in period 2 profits from setting a capacity constraint $K$ and then choosing $p_{2}$ optimally, rather than setting $p=A / 2$ and selling the 
unconstrained optimum $Q_{2}^{\prime}$. The loss function takes on a value of zero for all $K>Q_{2}^{\prime}$, since then the capacity constraint does not bind in period 2 and the firm can just sell $Q_{2}^{\prime}$.

Period 2 profits, $\pi_{2 c}$, are strictly increasing in $Q_{2 c}-Q_{2}^{\prime}$ whenever $Q_{2 c}<$ $Q_{2}^{\prime}$. This means that for any $K \leq Q_{2}^{\prime}$, the firm maximizes period 2 profits by setting $Q_{2 c}$ as close as possible to the unconstrained optimum $Q_{2}^{\prime}: Q_{2 c}=K$. Any higher price would decrease $Q_{2 c}$ further away from $Q_{2}^{\prime}$, and any lower price would generate strictly positive excess demand.

By (12) and $q_{2 c}=K$, the firm with capacity constraint $K$ will set period 2 price

$p_{2}=A-\frac{K}{x}\left\{(1-\alpha)\left[\frac{1}{1-(1-\alpha) C x}\right]\left[1+\left(\frac{\alpha C x}{1-C E[X \mid X \geq x]}\right)\right]+\alpha\left(\frac{1}{1-C E[X \mid X \geq x]}\right)\right\}^{-1}$

and earn period 2 profits

$\pi_{2 c}=A K-\frac{K^{2}}{x}\left\{(1-\alpha)\left[\frac{1}{1-(1-\alpha) C x}\right]\left[1+\left(\frac{\alpha C x}{1-C E[X \mid X \geq x]}\right)\right]+\alpha\left(\frac{1}{1-C E[X \mid \geq X]}\right)\right\}$

For any $K \leq Q_{2}^{\prime}$, the loss function $\lambda_{2}(K)$ is decreasing quadratically in $K$. It takes on a value of zero at $K=Q_{2}^{\prime}$, as does its derivative with respect to $K$.

The firm chooses $K$ to maximize $\pi=\pi_{1 c}+\delta \pi_{2 c}$, which is equivalent to minimizing $\lambda_{1}(K)+\delta \lambda_{2}(K)$. For the optimal $K$, we cannot have $K<Q_{1 u}$, since then marginally increasing $K$ would decrease both $\lambda_{1}(K)$ and $\lambda_{2}(K)$. Similarly, we cannot have $K>Q_{2}^{\prime}$, since then marginally decreasing $K$ would decrease $\lambda_{1}(K)$ while leaving $\lambda_{2}(K)$ unchanged. This implies $Q_{1 u} \leq K \leq Q_{2}^{\prime}$. For any such $K, \lambda_{1}(K)$ is increasing in $K$ while $\lambda_{2}(K)$ is decreasing in $K$.

The derivative of $\lambda_{1}(K)$ is zero at $K=Q_{1 u}$, while the derivative of $\lambda_{2}(K)$ is strictly negative. Also, the derivative of $\lambda_{2}(K)$ is zero at $K=Q_{2}^{\prime}$, while the derivative of $\lambda_{1}(K)$ is strictly positive. This implies that to minimize the weighted sum of $\lambda_{1}(K)$ and $\lambda_{2}(K)$, the firm must choose $Q_{1 u}<K<Q_{2}^{\prime}$.

Setting $p_{1}=A / 2$ would give $q_{1 c}=Q_{1 u}$, and setting $p_{2}=A / 2$ would give $q_{2 c}=Q_{2}^{\prime}$. Quantity demanded is decreasing in price, so $Q_{1 u}<Q_{1 c}$ implies $p_{1}<A / 2$ and $Q_{2 c}<Q_{2}^{\prime}$ implies $p_{2}>A / 2$. 
The result shows that if the firm can profitably use a capacity constraint, and if $K$ is chosen optimally, then the constraint will exactly bind in both periods. Naive consumers will then observe that period 1 quantity sold equals capacity. Because they are naive, these consumers do not conclude from the firm's equilibrium strategy that excess demand must be zero. Instead, they just infer that quantity demanded is greater than or equal to capacity.

In short, naive consumers will be fooled into believing demand is greater than it actually is. Their expectation $E[X]$ will be replaced in the expression for demand by the conditional expectation $E[X \mid X \geq x]$. The fact that the capacity constraint exactly binds makes this conditional expectation as high as possible. The new, more positive beliefs will increase consumers' willingness to pay in period 2, both through the direct and indirect effect described before Proposition 1.

Using a capacity constraint means that demand will always increase going from period 1 to period 2. However, using a capacity constraint also means the firm cannot fully taking advantage of this increased demand, because it cannot increase output. The firm would like to sell strictly more in period 2 than in period 1, but the capacity constraint limits quantity sold to $K$.

The optimal choice of $K$ reflects a trade-off between period 1 and period 2 profits. The firm will choose a capacity level between the optimal period 1 quantity and the optimal period 2 quantity, where the exact value of $K$ depends on how the firm weighs profits in each period.

Since quantity sold is the same in each period, prices must increase over time. Moreover, the period 1 price is lower than that which would maximize period 1 profits without a constraint, $p=A / 2$, while the period 2 price is higher.

Because the capacity constraint binds in both periods, naive consumers never discover they have been fooled. They observe that quantity sold equals capacity in period 2, which again leaves them unable to infer the true level of demand. Unlike in period 1, the reason the capacity constraint binds has nothing to do with influencing beliefs. The capacity constraint binds in period 2 because the firm would like to increase quantity sold in response to the increased demand, but it is prevented from doing so by its capacity constraint.

The next proposition describes under what conditions the firm will impose a capacity constraint.

Proposition 3. The firm will impose a capacity constraint whenever demand 
is lower than expected, but will not impose a capacity constraint if demand is sufficiently high.

Specifically, there exists a critical value $x^{\prime}$, with $E[X]<x^{\prime}$, such that $\left.\pi_{c}\right\rangle$ $\pi_{u}$ for all $x \in\left[0, x^{\prime}\right)$. There also exists a critical value $x^{\prime \prime}$, with $x^{\prime}<x^{\prime \prime}<1$, such that $\pi_{c}<\pi_{u}$ for any $x \in\left(x^{\prime \prime}, 1\right]$.

Proof. Suppose $x<E[X]$, and the firm does not impose a capacity constraint. By Proposition 1, it will then set $p_{1}=p_{2}=A / 2$. Quantity sold is then $Q_{1 u}$ given by (10) and $Q_{2 u}$ given by (11). Per period profits are $\pi_{1 u}$ given by (7) and $\pi_{2 u}$ by (8). We have $q_{2 u}<q_{1 u}$, since $x<E[x]$, so $Q_{2 u}<Q_{1 u}$. Quantity sold in period 2 is less than quantity sold in period 1.

Now say the firm imposes a capacity constraint $K=Q_{1 u}$, and sets $p_{1}=$ $p_{2}=A / 2$. Period 1 demand and quantity sold is then the same as without a constraint, $Q_{1 c}=Q_{1 u}$, so $\pi_{1 c}=\pi_{1 u}$. Quantity demanded equals capacity, $q_{1 c}=K$, so naive consumers update their beliefs from $E[X]$ to $E[X \mid X \geq x]$. This implies period 2 demand is higher than period 1 demand, $q_{2 c}>q_{1 u}$, as $q_{2 c}$ is given by (12) and $E[X \mid X \geq x]>E[X]$. At price $p_{2}=A / 2$, the firm can sell quantity $Q_{2 c}$, with $Q_{1 c}<Q_{2 c} \leq K$. Combined with $Q_{2 u}<Q_{1 u}$ and $Q_{1 u}=Q_{1 c}$, this implies $Q_{2 u}<Q_{2 c}$ Profits are therefore strictly higher than $\pi_{u}$, and the firm would prefer to use a capacity constraint.

If $x=E[X]$, then the optimal price without a capacity constraint is still $p_{1}=p_{2}=A / 2$. Naive consumers' expectation of $X$ turns out to be correct, so period 2 demand is just equal to period 1 demand. Quantity sold is $Q_{1 u}=Q_{2 u}$, given by (11).

If the firm imposes a capacity constraint $K=Q_{1 u}$ and sets $p_{1}=A / 2$, then again we have $\pi_{1 c}=\pi_{1 u}$. Just as above, we have $q_{2 c}>q_{1 c}$ because $E[X \mid X \geq x]>x$. Setting $p_{2}=A / 2$ yields strictly positive excess demand in period 2 , so the firm can charge $p_{2}$ marginally higher than $A / 2$ and still sell $Q_{2 c}=Q_{1 c}=K$. Period 2 profits are therefore strictly higher than $\pi_{2 u}$, and the firm would prefer to use a capacity constraint.

Profits are continuous in $x$, so the same conclusion must hold for $x>E[x]$, if $x$ is sufficiently close to $E[x]$. This implies there is a critical value $x^{\prime}$, with $E[X]<x^{\prime}$, such that $\pi_{c}>\pi_{u}$ for all $x \in\left[0, x^{\prime}\right)$.

Now suppose $x=1$. Then $E[X \mid X \geq x]=x$, so period 2 demand is the same regardless of whether the firm imposes a capacity constraint: comparing (6) and (12), we have $q_{2 c}=q_{2 u}$. A capacity constraint just constrains $Q_{2 c} \leq$ $K$, so we must have $\pi_{2 u} \geq \pi_{2 c}$. By Proposition 2, we have $Q_{1 c}=K$, where the proof showed that $K>Q_{1 u}$. Period 1 quantity sold is strictly greater 
than the unconstrained optimum, which implies $\pi_{1 u}>\pi_{1 c}$. This implies $\pi_{u}>\pi_{c}$, and the firm will prefer not to use a capacity constraint.

Finally, suppose $x<1$. Again by Proposition 2, if $\pi_{c}>\pi_{u}$, then the firm will set $p_{1}$ and $p_{2}$ such that $Q_{1 c}=Q_{2 c}=K$. Moreover, $Q_{1 u}<K<Q_{2}^{\prime}$, with $Q_{2}^{\prime}$ given by (12) evaluated at $p_{2}=A / 2$. Recall that $Q_{2}^{\prime}$ is the optimal quantity sold if naive consumers held beliefs $E[X \mid X \geq x]$ and the firm was not capacity constrained.

As $x$ tends to $1, E[X \mid X \geq x]$ tends to 1 as well. Demand is continuous in $x$, and $q_{2 c}=q_{2 u}$ for $x=1$. This means that $q_{2 c}$ must tend to $q_{2 u}$.

In the limit, we must therefore have $\pi_{2 c} \leq \pi_{2 u}$. Since $\pi_{1 u} \geq \pi_{1 c}$, the only way that $\pi_{c} \geq \pi_{u}$ can hold for $x$ sufficiently close to 1 , is that both $\pi_{1 u}-\pi_{1 c}$ and $\pi_{2 u}-\pi_{2 c}$ tend to zero as $x$ tends to 1 .

For $\pi_{2 u}-\pi_{2 c}$ to tend to zero, $Q_{2 c}$ must tend to the unconstrained optimum $Q_{2}^{\prime}$. But $Q_{2 c}=K$, so $K$ must tend to $Q_{2}^{\prime}$ as well. From the proof of Proposition 2, we know that $Q_{1 u}<Q_{2}^{\prime}$, so if $K$ tends to $Q_{2}^{\prime}$, we have $K>Q_{1 u}$. But by $Q_{1 c}=K$, this implies period 1 output is strictly higher than the unconstrained optimum, and in the limit $\pi_{1 u}>\pi_{1 c}$. For $x$ sufficiently close to 1 , profits are strictly higher without a capacity constraint.

The result shows that consumers will observe a sell-out when demand is low, not when it is high. This is a very different conclusion than one would likely reach if capacity was exogenous, or if the firm was uninformed about demand. In that case, it would seem intuitive that a sell-out be associated with high demand.

What drives the results is that the firm imposes a capacity constraint with the express purpose of fooling naive consumers. Naive consumers do associate a sell-out with high demand, because they ignore the firm's strategic behavior. It is precisely the fact that naive consumers make this inference that gives the firm an incentive to fool them when demand is low.

The firm's decision to impose a capacity constraint generates a benefit, but also an implicit cost. The benefit of a capacity constraint is that it makes period 2 demand higher than it otherwise would have been. The discussion after Proposition 2 showed that imposing a capacity constraint causes demand to increase after period 1 . More to the point, it also ensures period 2 demand is higher than if the firm had not imposed a constraint: $q_{2 c}>q_{2 u}$.

The implicit cost of a capacity constraint is that the firm cannot increase 
output over time. Without a constraint, the firm can maximize period 2 profits by selling $Q_{2 u}$, which is the optimal output given demand $q_{2 u}$. If $x>E[x]$, then demand is higher in period 2 as naive consumers infer the true value of $x$, and quantity sold increases over time. With a capacity constraint, increasing quantity sold in this way is impossible. For certain values of $x$, a capacity constrained firm will be forced to sell less in period 2 than if it had not imposed the constraint: $Q_{2 c}<Q_{2 u}$.

Whether imposing a capacity constraint increases profits depends on how the benefit compares to the possible cost. If demand is lower than expected, $x \leq E[x]$, then demand would decrease over time without a capacity constraint. There would then be no question of increasing output in period 2, and the firm would choose some $Q_{2 u} \leq Q_{1 u}$.

Proposition 2 states that a firm with a capacity constraint will choose $K$ greater than the unconstrained period 1 quantity sold: $K=Q_{1 c}>Q_{1 u}$. This constraint will not force the firm to sell less than $Q_{1 c}$ in period 2, so the implicit cost of a capacity constraint is zero. Its only effect is to increase period 2 demand and increase profits.

The cost and benefits of a capacity constraint are different if $x$ is close to 1. A capacity constraint will then not fool naive consumers by very much, since $E[X \mid X \geq x]$ is close to $x$. Imposing a capacity constraint therefore has little effect on period 2 demand. In contrast, a large value of $x$ means that a firm without a constraint would certainly expand output in period 2, which leads to $Q_{2 u}>Q_{2 c}$. In this case, the cost of a capacity constraint will exceed the benefit. ${ }^{12}$

An important reason behind Proposition 3, as well as for the other results in this paper, is that aggregate demand is positively correlated across periods. I make the stark assumption that it is perfectly correlated, so that the number of consumers in the market is the same in each period. It is positive correlation that allows consumers who see a sell-out in period 1 to infer that demand will also be high in period 2, which in turn gives the firm an incentive to impose a capacity constraint.

The assumption of perfect correlation is not necessary for this type of mechanism to work, but it does simplify the analysis. If the number of consumers in the market could vary across periods, then an important issue

\footnotetext{
${ }^{12}$ Proposition 3 leaves open the question of whether the relationship between demand and the firm's incentive to impose a capacity constraint is monotonic. If so, the firm would impose a capacity constraint if and only if $x$ is below some critical value. I can show that this is the case if $C$ is small, but whether this holds more generally remains to be seen.
} 
would be whether the firm observes both periods' demand before imposing a capacity constraint. If not, then imposing a capacity constraint when period 1 demand is low would now carry a positive cost. The firm would have to take into account that period 2 demand could still be high, in which case expanding output would be important.

Proposition 3 also immediately implies the following corollary.

Corollary. The ex-ante probability that the firm will impose a capacity constraint is bounded below by $F(E[X])$. This probability is therefore strictly positive even in the limit as $C, \alpha$ or $\delta$ tend to zero.

This corollary shows there is a discontinuity in the firm's optimal strategy when some parameters equal zero. If $C=0, \alpha=0$ or $\delta=0$, then the situation reduces to that without social effects. There is then either no social utility, no naive consumers to fool, or the firm places no weight on period 2 profits. In such a case, a capacity constraint could never strictly increase profits.

The situation is very different if these parameters are strictly positive but small. As any one of them tends to zero, the range of $x$ for which a capacity constraint is profitable does not.

The intuition is that both the cost and benefit of a capacity constraint depend on the fraction of naive consumers and the strength of social effects. If there are few naive consumers, then imposing a capacity constraint will not increase period 2 demand by much over the unconstrained level. But having few naive consumers also means that expanding output is less of an issue, because demand does not change much over time. In the limit, the benefit of a capacity constraint tends to zero, but so does the cost.

The next proposition gives an explicit expression for $K$, the firm's optimal capacity constraint. The result shows that even though the firm's decision whether or not to use a capacity constraint is discontinuous in parameter values, the equilibrium prices and quantities are not.

Proposition 4. The optimal capacity constraint $K$ is given by

$$
K=\frac{x A(1+\delta)}{2 Z}
$$

where

$$
Z=\left\{\left(\frac{1}{1-C E[X]}\right)\left[(1-\alpha)\left(\frac{1-C(E[X]-\alpha x)}{1-C x(1-\alpha)}\right)+\alpha\right]\right\}^{-1}+
$$




$$
\delta\left\{\left(\frac{1}{1-C E[X \mid X \geq x]}\right)\left[(1-\alpha)\left(\frac{1-C(E[X \mid X \geq x]-\alpha x)}{1-C x(1-\alpha)}\right)+\alpha\right]\right\}^{-1} .
$$

$K$ is increasing in $\delta$. As $C$ or $\alpha$ tend to zero, $K$ tends to the unconstrained optimum $Q_{1 u}$ given by (10), and $p_{1}$ tends to $A / 2$.

Proof. Rearranging (5), Period 1 demand is given by

$$
q_{1}=\left(\frac{1}{1-C E[X]}\right)\left[(1-\alpha)\left(\frac{1-C(E[X]-\alpha x)}{1-C x(1-\alpha)}\right)+\alpha\right] x(A-p)
$$

If the firm sets a capacity constraint, then rearranging (12) shows period 2 demand is

$$
q_{2}=\left(\frac{1}{1-C E[X \mid X \geq x]}\right)\left[(1-\alpha)\left(\frac{1-C(E[X \mid X \geq x]-\alpha x)}{1-C x(1-\alpha)}\right)+\alpha\right] x(A-p)
$$

If the firm sets capacity constraint $K$, then $p_{1}$ and $p_{2}$ are such that $q_{1}=$ $q_{2}=K$. That is

$$
\begin{gathered}
p_{1}=A-\frac{K}{x}\left\{\left(\frac{1}{1-C E[X]}\right)\left[(1-\alpha)\left(\frac{1-C(E[X]-\alpha x)}{1-C x(1-\alpha)}\right)+\alpha\right]\right\}^{-1}, \\
p_{2}=A-\frac{K}{x}\left\{\left(\frac{1}{1-C E[X \mid X \geq x]}\right)\left[(1-\alpha)\left(\frac{1-C(E[X \mid X \geq x]-\alpha x)}{1-C x(1-\alpha)}\right)+\alpha\right]\right\}^{-1} .
\end{gathered}
$$

Total profits are then $\left(p_{1}+\delta p_{2}\right) K$, so

$$
\pi_{c}=A(1+\delta) K-\frac{Z}{x} K^{2}
$$

where $Z$ is given by (15). Profits are quadratic in $K$, and the first order condition gives

$$
K=\frac{x A(1+\delta)}{2 Z}
$$


Taking the derivative of $K$ with respect to $\delta$, this is positive if

$$
Z-(1+\delta) \frac{\partial Z}{\partial \delta}>0
$$

Looking at (16) and (17) and the fact that $q_{2}>q_{1}$ means that

$$
\begin{gathered}
\left\{\left(\frac{1}{1-C E[X]}\right)\left[(1-\alpha)\left(\frac{1-C(E[X]-\alpha x)}{1-C x(1-\alpha)}\right)+\alpha\right]\right\}^{-1}> \\
\left\{\left(\frac{1}{1-C E[X \mid X \geq x]}\right)\left[(1-\alpha)\left(\frac{1-C(E[X \mid X \geq x]-\alpha x)}{1-C x(1-\alpha)}\right)+\alpha\right]\right\}^{-1}
\end{gathered}
$$

Comparing this with (15), it follows that $(1+\delta) \frac{\partial Z}{\partial \delta}<Z$ so that $K$ is increasing in $\delta$.

If $\pi_{c}>\pi_{u}$, then by Proposition 2 we have $Q_{1 u}<K$. From (15) and (18), $K$ is continuous in all parameters. The limit of $K$ as $C$ or $\alpha$ tend to zero is therefore equal to (18) evaluated at $C=0$ or $\alpha=0$. I now show that this is equals to $Q_{1 u}$ evaluated at these parameter values.

Recall that $Q_{1 u}$ was given by (10),

$Q_{1 u}=\left\{(1-\alpha)\left[\frac{1}{1-(1-\alpha) C x}\right]\left[1+\left(\frac{\alpha C x}{1-C E[X]}\right)\right]+\alpha\left(\frac{1}{1-C E[X]}\right)\right\} x\left(\frac{A}{2}\right)$.

Setting $\alpha=0$ in (15) gives $Z=(1+\delta)(1-C x)$, which implies $K=$ $x A / 2(1-C X)$. Comparing with the above expression, this is the same as $Q_{1 u}$ evaluated at $\alpha=0$. Setting $C=0$ in (15) gives $Z=(1+\delta)$, which implies $K=x A / 2$. Again, comparing with the above expression shows this is just $Q_{1 u}$ evaluated at $C=0$.

To complete the proof, note that $Q_{1 u}$ is the quantity demanded corresponding to $p_{1}=A / 2$. If $q_{1 c}=K$ tends to $Q_{1 u}$, then the firm which imposes a capacity constraint must charge price $p_{1}$ that tends to $A / 2$.

The result shows that the firm's optimal capacity constraint $K$ approaches the unconstrained optimal quantity sold, as social effects or the fraction of naive consumers becomes small. For small values of $\alpha$ or $C$, fooling naive consumers has little effect on period 2 demand. It is then quite intuitive that the firm choose $K=Q_{1 c}$ close to $Q_{1 u}$. 
The optimal capacity constraint increases as the firm becomes more patient. By Proposition 2, the firm chooses a value of $K$ between that which would maximize period 1 profits, and the higher level that would maximize period 2 profits. The firm's choice of $K$ therefore reflects a trade-off between profits in these two periods. As the firm becomes more patient, it places more weight on period 2 profits and it will choose a higher capacity constraint.

The final result follows in a similar vein, showing that a patient firm is more likely to impose a capacity constraint.

Proposition 5. The ex-ante probability that the firm will impose a capacity constraint is strictly increasing in $\delta$. Specifically, let $S_{\delta} \in[0,1]$ be the set of $x$ for which $\pi_{c}>\pi_{u}$, for given $\delta$. Then $\delta_{1}<\delta_{2}$ implies $S_{\delta_{1}} \subset S_{\delta_{2}}$.

Proof. Fix $\delta=\delta_{1}<1$, and consider some value of $x$ for which $\pi_{c} \geq \pi_{u}$. If the firm does not impose a capacity constraint, then $\pi_{u}=\pi_{1 u}+\delta \pi_{2 u}$, with $\pi_{1 u}$ given by (7) and $\pi_{2 u}$ given by (8). If the firm imposes the optimal capacity constraint $K$, then $\pi_{c}=\pi_{1 c}+\delta \pi_{2 c}$, with $\pi_{1 c}$ given by (13) and $\pi_{2 c}$ given by (14).

We have $\pi_{c}-\pi_{u}=\delta_{1}\left(\pi_{2 c}-\pi_{2 u}\right)-\left(\pi_{1 u}-\pi_{1 c}\right) \geq 0$. By Proposition 2, we know that $Q_{1 u}<K$. Since $Q_{1 u}$ maximizes period 1 profits without a capacity constraint, it follows that $\pi_{1 u}>\pi_{1 c}$. The only way that $\pi_{c} \geq \pi_{u}$ can hold is if $\pi_{2 c}>\pi_{1 u}$. That implies both terms in brackets in the expression for $\pi_{c}-\pi_{u}$ are strictly positive.

Now consider some $\delta_{2}>\delta_{1}$, and let $\delta=\delta_{2}$. By Proposition 1, the optimal prices without a capacity constraint remains $p_{1}=p_{2}=A / 2$, so $\pi_{1 u}$ and $\pi_{2 u}$ are the same as when $\delta=\delta_{1}$. If the firm imposes a capacity constraint with $\delta=\delta_{2}$, it can always choose the same values of $K, p_{1}$ and $p_{2}$ as when $\delta=\delta_{1}$. By so doing, it earns the same per period profits $\pi_{1 c}$ and $\pi_{2 c}$. But now $\delta_{2}>\delta_{1}$, which implies $\pi_{c}-\pi_{u}=\delta_{2}\left(\pi_{2 c}-\pi_{2 u}\right)-\left(\pi_{1 u}-\pi_{1 c}\right)>0$. The firm will therefore use a capacity constraint for this value of $x$.

To complete the proof, it is sufficient to show that there exists some $x$ such that $\pi_{c}=\pi_{u}$ for $\delta=\delta_{1}$. In that case, $S_{\delta_{1}}$ will be a proper subset of $S_{\delta_{2}}$. This is indeed the case. Profits are continuous in $x$, and we have $\pi_{c}>\pi_{u}$ for $x \leq E[x]$ and $\pi_{c}<\pi_{u}$ for $x$ close to 1 . Moreover, $X$ has full support on $[0,1]$. So for any $\delta$, by the Intermediate Value Theorem there must be some $x$ with $E[x]<x<1$ for which $\pi_{c}=\pi_{u}$.

The intuition behind the result is that the decision to impose a capacity constraint is based on a trade-off between period 1 and period 2 profits. A 
capacity constraint has no immediate effect on demand, and so it cannot increase period 1 profits above the unconstrained level. On the other hand, a capacity constraint can be optimal if its positive impact on period 2 demand is sufficiently large. A patient firm places a higher weight on period 2 profits, and so finds a capacity constraint more attractive.

\section{Literature on Naive Consumers}

A key factor driving the results in this paper is the presence of naive consumers, who are unable to directly infer anything about demand by observing the actions taken by the firm. Naive consumers are not irrational, and in many ways are actually quite sophisticated. They understand the equilibrium strategies of other consumers, and they maximize their expected utility. Naive consumers also use Bayes' rule when updating their beliefs about aggregate demand, taking into account the price and quantity sold that they observe. These consumers are only naive in the sense that they do not realize that the firm acts strategically, that its equilibrium actions will depend on the information it has about demand.

This approach is consistent with evidence suggesting that people may not fully realize how other people's actions depend on their private information. One such case is the winner's curse in common value auctions. This can occur if a bidder does not understand that others will only place low bids, allowing him to win, if they have negative private information about the object's value. Another is where people may still trade in situations with asymmetric information, where Bayesian Nash equilibrium predicts no trade should occur. An uninformed buyer may not appreciate that the seller's decision to accept an offer will depend on his private information about the good's value, so that he unwittingly ends up buying a "lemon" (Holt and Sherman 1994).

Eyster and Rabin (2005) consider naive consumers in games of incomplete information, and define what they call a cursed equilibrium. A player is cursed if he correctly predicts the distribution of equilibrium actions, but underestimates the extent to which these actions correspond to other players' types. The situation here where all consumers are naive corresponds to their fully cursed equilibrium, where no player infers anything about demand from the firm's choice of price or capacity. The situation where only some consumers are naive is somewhat different from what they call a partially 
cursed equilibrium. There, all consumers underestimate the extent to which equilibrium actions are related to private information, but only to a certain degree.

A related approach is Jehiel (2005)'s concept of analogy based expectation equilibrium, where each player groups others into analogy classes and expects them to act as the average player in that class. In a situation with incomplete information, for a certain analogy partition, this solution concept is equivalent to that of fully cursed equilibrium (Jehiel and Koessler 2008). Behavioral equilibrium, as proposed by Esponda (2008), adds the requirement that naive players' beliefs must be consistent with the information revealed from the outcome of equilibrium play. The results here can be seen in this light, as naive consumers' whose beliefs are manipulated never discover that they have been fooled.

An important feature of this paper is that a capacity constraint can prevent naive consumers from inferring the true level of demand. This relates to recent work in industrial organization on how firms may try to hide information from boundedly rational consumers. Gabaix and Laibson (2006) show firms may use shrouding to fool naive consumers who are not aware that it charges add-on prices. Spiegler (2006) considers consumers who evaluate a product by only sampling quality in one dimension, and shows firms may obfuscate by randomizing over quality in other dimensions. Chioveanu and Zhou (2009) and Piccione and Spiegler (2009) look at price competition with framing, where firms choose both prices and the frames in which prices are presented. By randomizing over both frames and prices, the firm can prevent consumers from making price comparisons.

These papers all examine why a firm may want to prevent consumers from learning about its own prices or products, or those of its competitors. What I show is related but has a slightly different flavor: that a firm may want to prevent consumers from learning about each others' actions.

\section{Conclusion}

This paper has shown how a firm can use a capacity constraint to manipulate consumer beliefs about aggregate demand. Imposing a capacity constraint and setting a price just low enough for it to bind can fool naive consumers, who just infer that quantity demanded must be greater than or equal to capacity. Through social effects, this increases the demand of naive consumers 
in the following period. Social effects also mean this will increase the willingness to pay of all other consumers, because they know naive consumer demand has increased.

An important assumption throughout has been that when a capacity constraint binds, consumers are unable to observe the extent of excess demand. For future work, it would be interesting to apply this idea to a model where consumers choose sequentially and there is a possibility of an information cascade. By imposing a capacity constraint, a firm could influence the extent to which consumers observe the actions of those who have chosen earlier, and therefore their inference about each others' private information. The question would then be whether imposing a capacity constraint could help trigger a positive cascade, or help sustain one which has already started.

\section{References}

Banerjee, A. V. (1992, August). A simple model of herd behavior. The Quarterly Journal of Economics 107(3), 797-817.

Basu, K. (1987). Monopoly, quality uncertainty and 'status' goods. International Journal of Industrial Organization 5(4), 435-446.

Becker, G. S. (1974, Nov.-Dec.). A theory of social interactions. Journal of Political Economy 82(6), 1063-93.

Becker, G. S. (1991, October). A note on restaurant pricing and other examples of social influences on price. Journal of Political Economy $99(5), 1109-16$.

Bikhchandani, S., D. Hirshleifer, and I. Welch (1992, October). A theory of fads, fashion, custom, and cultural change in informational cascades. Journal of Political Economy 100(5), 992-1026.

Chioveanu, I. and J. Zhou (2009, September). Price competition and consumer confusion. MPRA Paper 17340, University Library of Munich, Germany.

DeGraba, P. (1995, Summer). Buying frenzies and seller-induced excess demand. RAND Journal of Economics 26 (2), 331-342.

Denicolo, V. and P. Garella (1999, Spring). Rationing in a durable goods monopoly. RAND Journal of Economics 30(1), 44-55. 
DeSerpa, A. C. and R. L. Faith (1996, October). "Bru-u-u-uce": The simple economics of mob goods. Public Choice 89(1-2), 77-91.

Esponda, I. (2008, September). Behavioral equilibrium in economies with adverse selection. American Economic Review 98(4), 1269-91.

Eyster, E. and M. Rabin (2005, September). Cursed equilibrium. Econometrica 73(5), 1623-1672.

Gabaix, X. and D. Laibson (2006, May). Shrouded attributes, consumer myopia, and information suppression in competitive markets. The Quarterly Journal of Economics 121(2), 505-540.

Holt, C. A. and R. Sherman (1994, June). The loser's curse. American Economic Review 84(3), 642-52.

Jehiel, P. (2005, August). Analogy-based expectation equilibrium. Journal of Economic Theory 123(2), 81-104.

Jehiel, P. and F. Koessler (2008, March). Revisiting games of incomplete information with analogy-based expectations. Games and Economic Behavior 62(2), 533-557.

Karni, E. and D. Levin (1994, August). Social attributes and strategic equilibrium: A restaurant pricing game. Journal of Political Economy 102(4), 822-40.

Katz, M. L. and C. Shapiro (1985, June). Network externalities, competition, and compatibility. American Economic Review 75 (3), 424-40.

Leibenstein, H. (1950, May). Bandwagon, snob, and Veblen effects in the theory of consumers demand. The Quarterly Journal of Economics 64(2), 183-207.

Nocke, V. and M. Peitz $(2007,07)$. A theory of clearance sales. Economic Journal 117(522), 964-990.

Piccione, M. and R. Spiegler (2009). Framing competition. Mimeo, LSE and $U C L$.

Spiegler, R. (2006, June). Competition over agents with boundedly rational expectations. Theoretical Economics 1(2), 207-231. 